\title{
Reprodutibilidade do Teste de 1-RM \\ em Indivíduos com Doença Arterial Obstrutiva Periférica
}

\section{Reproducibility of the 1-RM Test in Individuals With Peripheral Obstructive Arterial Disease}

Raphael Mendes Ritti Dias Gabriel Grizzo Cucato² Lucas Caseri Câmara ${ }^{3}$ Nelson Wolosker ${ }^{3}$

1. Escola Superior de Educação Física, Universidade de Pernambuco - Recife, PE.

2. Escola de Educação Física e Esportes, Universidade de São Paulo - São Paulo, SP.

3. Faculdade de Medicina, Universidade de São Paulo - São Paulo, SP.

\section{Endereço para correspondência:} Prof. Raphael Mendes Ritti Dias Escola Superior de Educação Física Universidade de Pernambuco Rua Arnóbio Marques, 310 - Santo Amaro

50100-300 - Recife, PE, Brasil E-mail: raphael.dias@upe.br

\section{RESUMO}

Introdução: Indivíduos com doença arterial obstrutiva periférica (DAOP) apresentam atrofia muscular e redução da força de membros inferiores que, por sua vez, estão associadas às limitações na capacidade funcional. Consequentemente, a mensuração da força muscular pode ser útil na identificação dos níveis de força muscular e para monitorar as alterações na força em programas de intervenção. Objetivo: Analisar a reprodutibilidade do teste de uma repetição máxima (1-RM) em indivíduos com DAOP. Métodos: Fizeram parte da amostra 26 indivíduos com DAOP e sintomas de claudicação intermitente. Os indivíduos realizaram quatro sessões de teste de 1-RM no exercício extensão de joelhos unilateral, no membro com maior índice tornozelo-braço (ITB) e no membro com menor ITB. Resultados: A força máxima aumentou significantemente da primeira para a segunda sessão de teste nos dois membros ( $4,1 \pm 14,3 \%$ na perna com menor ITB e 5,9 $\pm 13,1 \%$ na perna com maior ITB). A análise dos limites de concordância revelou que, nos dois membros, as maiores diferenças médias e os maiores limites de concordância foram observados quando comparadas as sessões 1 e 2. Conclusão: A medida acurada da força máxima usando o teste de 1-RM, no exercício extensão de joelhos, em indivíduos com DAOP e sintomas de claudicação intermitente, é obtida quando duas sessões de testes de 1-RM são realizadas.

Palavras-chave: força muscular, claudicação intermitente, reprodutibilidade dos testes.

\section{ABSTRACT}

Introduction: Patients with peripheral obstructive arterial disease (PAD) present muscle atrophy and reduced strength in the legs which have been associated with impairment in physical function. Consequently, the assessment of leg strength can be useful for identifying muscle weakness and analyzing the efficacy of intervention programs for patients with PAD. Objective: To assess the reliability of the 1 -RM test in patients with PAD. Methods: The sample was composed of twenty-six patients with PAD and symptoms of mild intermittent claudication. Patients performed the 1-RM tests in the knee extension exercise in the leg with lower ankle brachial index (ABI) and in the leg with higher $A B I$. This protocol was repeated in four sessions to assess the reliability of the maximum strength measurements. Results: Maximal strength increased significantly from session 1 to session 2 in both legs $(4.1 \pm 14.3 \%$ in the leg with lower $A B I$ and $5.9 \pm 13.1 \%$ in the leg with higher $A B I)$. The analysis of agreement revealed that higher bias and higher limits of agreement were observed in both legs when sessions 1 and 2 were compared. Conclusion: Reliable assessment of strength using 1-RM test in knee extension exercise in patients with PAD and symptoms of intermittent claudication is obtained when 2 test sessions are performed.

Keywords: muscle strength, intermittent claudication, reproducibility of results. 


\section{INTRODUÇÃO}

Indivíduos com doença arterial obstrutiva periférica (DAOP) apresentam atrofia muscular (1) e redução de força nos membros inferiores ${ }^{(2,3)}$, que tem sido associada com as limitações na capacidade funcional desses indivíduos. Consequentemente, a avaliação da força muscular em indivíduos com DAOP tem sido útil para identificar os níveis de força muscular e para monitorar as alterações nessa variável ao longo de programas de intervenção(2-7). Além disso, em indivíduos com disfunções ortopédicas limitantes para a caminhada, como os amputados, e em indivíduos que apresentam a doença nos dois membros inferiores, o teste de força tem sido indicado por fornecer indicativo da força muscular em cada membro individualmente.

Para a avaliação da força muscular é importante que o método utilizado apresente boa reprodutibilidade ${ }^{(8)}$. Em indivíduos com DAOP o método que tem apresentado os melhores coeficientes de reprodutibilidade é o dinamômetro isocinético(2). Entretanto, devido ao seu custo elevado, a avaliação da força muscular com esse equipamento não tem sido incorporada nas rotinas de avaliação de indivíduos com DAOP. Uma alternativa mais viável economicamente para avaliação da força é o teste de 1-RM, que embora não permita controlar a velocidade do movimento como o dinamômetro isocinético, apresenta algumas vantagens, tais como a facilidade de execução, a especificidade com o treinamento de força e o baixo estresse cardiovascular ${ }^{(9)}$. Além disso, o teste de 1-RM tem apresentado altos coeficientes de reprodutibilidade tanto em jovens, como em idosos ${ }^{(10)}$.

Apesar dessas vantagens, até o presente momento, não existem relatos da utilização, bem como da reprodutibilidade do teste de 1-RM em indivíduos com DAOP, o que é de fundamental importância para que esse teste seja incorporado às rotinas de avaliação desses indivíduos. Assim, o objetivo deste estudo foi avaliar a reprodutibilidade do teste de 1-RM em indivíduos com DAOP.

\section{MÉTODOS}

\section{Amostra}

Este estudo foi aprovado pelo Comitê de Ética em Pesquisa com Seres Humanos da instituição do primeiro autor (processo 1.370/05). Previamente ao início das coletas, cada indivíduo foi informado dos riscos e benefícios envolvidos no estudo e assinaram o termo de consentimento livre e esclarecido.

Sessenta indivíduos matriculados em ambulatório especializado de cirurgia vascular realizaram triagem para participação no estudo. A triagem foi composta de exames clínicos e obtenção do histórico médico. Os exames clínicos consistiram de avaliação de limitações ortopédicas, medidas da pressão arterial de tornozelo e de braço para obtenção do índice tornozelo-braço (ITB) ${ }^{(11)}$ e realização de teste máximo em esteira ergométrica ${ }^{(12)}$. O histórico médico envolveu questões referentes aos níveis de atividade física, tabagismo e doenças associadas.

Os indivíduos eram incluídos no estudo se eles apresentassem o estágio II da DAOP de acordo com os critérios de Fontaine ${ }^{(13)}$, sintomas de claudicação intermitente por pelo menos seis meses, ITB em repouso $\leq 0,90$ em um ou dois membros e tolerância ao exercício limitada pelos sintomas de claudicação. Os indivíduos em que a medida do ITB não pôde ser obtida, que apresentavam tolerância ao exercício limitada por outros fatores que não a claudicação e que apresentavam respostas anormais no eletrocardiograma ou da pressão arterial durante o teste ergométrico, não foram incluídos no estudo. No total, 26 indivíduos foram considerados elegíveis para esse estudo.

\section{Estratificação dos fatores de risco}

A atividade física foi autorrelatada e os indivíduos foram classificados em inativos (<150 min/sem de atividade física de lazer ou de transporte) ou ativos ( $\geq 150 \mathrm{~min} / \mathrm{sem}$ de atividade física de lazer ou de transporte). O tabagismo foi autorrelatado e os indivíduos foram classificados em tabagistas (fumantes atuais diários e ocasionais) ou ex-fumantes (que pararam de fumar há pelo menos seis meses). A presença de hipertensão foi identificada pelo diagnóstico prévio, o uso de medicação anti-hipertensiva ou valores de pressão arterial sistólica e diastólica $\geq$ $140 \mathrm{mmHg}$ e $90 \mathrm{mmHg}$, respectivamente ${ }^{(14)}$. O diabetes foi identificado pelo diagnóstico prévio, pelo uso de medicação anti-hipertensiva ou pela glicemia de jejum $\geq 126 \mathrm{mg} / \mathrm{dl}^{(15)}$. A obesidade foi identificada pelo índice de massa corporal $\geq 30,0 \mathrm{~kg} / \mathrm{m}^{2}$. A presença de doença cardiovascular foi identificada pelo histórico de doença da arterial coronária, insuficiência cardíaca, ou realização prévia de cirurgia de revascularização.

\section{Teste de 1-RM}

A força máxima foi obtida usando o teste de 1-RM no exercício extensão de joelhos. Para tanto, foi utilizado equipamento de força (Biodelta® ${ }^{\oplus}$, Brasil). O teste foi realizado em quatro sessões, em dias distintos, intervalados por pelo menos 72 horas. Os testes foram realizados unilateralmente no membro em que a doença era mais grave (menor ITB) e no membro em que a doença era menos grave (maior ITB). Na primeira sessão de teste, a ordem dos membros foi obtida aleatoriamente, e depois mantida para as demais sessões.

Em cada sessão de teste, era realizada, inicialmente, uma série de aquecimento (6-10 repetições) com aproximadamente 50\% da carga que seria utilizada na primeira tentativa. Após dois minutos era iniciado o teste. Os indivíduos eram orientados a tentar realizar duas execuções completas. Uma execução completa era considerada quando os indivíduos conseguiam estender o joelho até uma marca, posicionada a aproximadamente $85^{\circ}$ de amplitude do movimento. Cada indivíduo tinha cinco tentativas para alcançar a carga referente a 1-RM. O intervalo de recuperação entre as tentativas e entre os membros variou de um a dois minutos ${ }^{(16)}$. A carga referente a 1-RM foi definida como a carga que o indivíduo conseguia realizar apenas uma execução completa ${ }^{(17)}$.

\section{Análise estatística}

Estatística descritiva, por meio de média e desvio padrão, foi utilizada para apresentação dos resultados. O coeficiente de correlação intraclasse (ICC) foi utilizado para analisar a correlação entre a carga máxima obtida nas quatro sessões de familiarização. A análise de variância de dois fatores para medidas repetidas, utilizando como fatores o membro (membro com menor ITB e membro com maior ITB) e as sessões (sessão 1, sessão 2, sessão 3 e sessão 4) foram utilizadas para comparar as alterações na força em cada membro ao longo das sessões. Quando verificado efeito significante, foi utilizado o teste post hoc de Newman-Keuls para identificação das diferenças. A análise de Bland-Altman foi utilizada para identificar as diferenças médias e os limites de concordância entre as sessões de teste. $O$ nível de significância adotado foi de $P<0,05$.

\section{RESULTADOS}

As características clínicas dos indivíduos incluídos no estudo são apresentadas na tabela 1. A maioria dos indivíduos era de idosos, não fumantes, hipertensos e com excesso de peso.

Não foram observadas lesões durante o teste de 1-RM em nenhuma das sessões. Os valores de força máxima, em cada membro, nas quatro sessões de teste, estão apresentados na tabela 2.

Em ambos os membros foram observados valores elevados de ICC. Os valores de ICC foram de 0,99 e 0,98 para os membros com menor e com maior ITB, respectivamente.

A perna com menor ITB tendeu a apresentar menores valores de força do que na perna com maior ITB $(P=0,10)$. A carga de 1-RM aumentou significantemente da sessão 1 para a sessão 2 nos dois membros $(4,1 \pm 14,3 \%$ no membro com menor ITB e $5,9 \pm 13,1 \%$ no membro 
Tabela 1. Características clínicas e antropométricas da amostra $(n=26)$.

\begin{tabular}{c|c}
\hline Idade (anos) & $64,1 \pm 8,7$ \\
\hline Peso (kg) & $72,1 \pm 15,2$ \\
\hline Estatura (cm) & $162 \pm 7$ \\
\hline Índice de massa corporal (kg.m²) & $27,5 \pm 5,0$ \\
\hline ITB na perna com menor ITB & $0,63 \pm 0,13$ \\
\hline ITB na perna com maior ITB & $0,72 \pm 0,18$ \\
\hline Distância de claudicação (m) & $506 \pm 308$ \\
\hline Distância máxima de caminhada (m) & $860 \pm 353$ \\
\hline Gênero (\% de homens) & 69 \\
\hline Inatividade física (\%) & 52 \\
\hline Tabagismo (\%) & 19 \\
\hline Hipertensão (\%) & 35 \\
\hline Diabetes melito (\%) & 30 \\
\hline Obesidade (\%) & 22 \\
\hline Doença cardíaca (\%) & \\
\hline
\end{tabular}

ITB - Índice tornozelo-braço

com maior ITB). Em ambos os membros, a carga máxima não foi diferente entre as sessões 2, 3 e 4 ( $P>0,05)$. Não foi observado efeito da interação membro*sessão $(P>0,05)$, indicando que os aumentos de força ao longo das sessões foram similares entre os membros.

A análise dos limites de concordância entre as sessões de familiarização está apresentada na figura 1. Em ambos os membros, as maiores diferenças médias e os maiores limites de concordância foram observados quando comparadas as sessões 1 e 2. Em ambos os membros
Tabela 2. Valores médios e desvio padrão da carga de 1-RM obtida nas quatro sessões de teste de força.

\begin{tabular}{|c|c|c|c|c|}
\hline & \multicolumn{2}{|c|}{ Perna com menor ITB } & \multicolumn{2}{|c|}{ Perna com maior ITB } \\
\hline & Média \pm DP & ICC & Média \pm DP & ICC \\
\hline Sessão 1 (kg) & $16,5 \pm 7,7$ & \multirow{4}{*}{0,99} & $16,3 \pm 7,6$ & \multirow{4}{*}{0,98} \\
\hline Sessão 2 (kg) & $17,7 \pm 7,9^{*}$ & & $17,3 \pm 8,1^{*}$ & \\
\hline Sessão 3 (kg) & $17,6 \pm 7,9^{*}$ & & $17,4 \pm 7,8^{*}$ & \\
\hline Sessão 4 (kg) & $17,8 \pm 7,9^{*}$ & & $17,5 \pm 8,1^{*}$ & \\
\hline
\end{tabular}

as menores diferenças médias foram observadas quando comparadas as sessões 2 e 3. Para o membro com menor ITB, os menores limites de concordância foram observados entre as sessões 3 e 4, enquanto que para o membro com maior ITB, os menores limites de concordância foram observados entre as sessões 2 e 3.

\section{DISCUSSÃO}

Para implementação de métodos de avaliação na prática clínica, é importante buscar métodos reprodutíveis, simples, de baixo custo e que não demandam muito tempo para avaliação ${ }^{(18)}$. Os resultados do presente estudo indicaram que o teste de 1-RM apresenta boa reprodutibilidade para avaliação da força máxima desde que sejam realizadas duas sessões de teste. Considerando que o teste de 1-RM é de simples de realização, de baixo custo e que não demanda tempo muito longo para sua realização (aproximadamente 10-15 min), é possível indicar que o teste de 1-RM atende pré-requisitos importantes para sua inclusão nas rotinas de avaliação de indivíduos com DAOP.

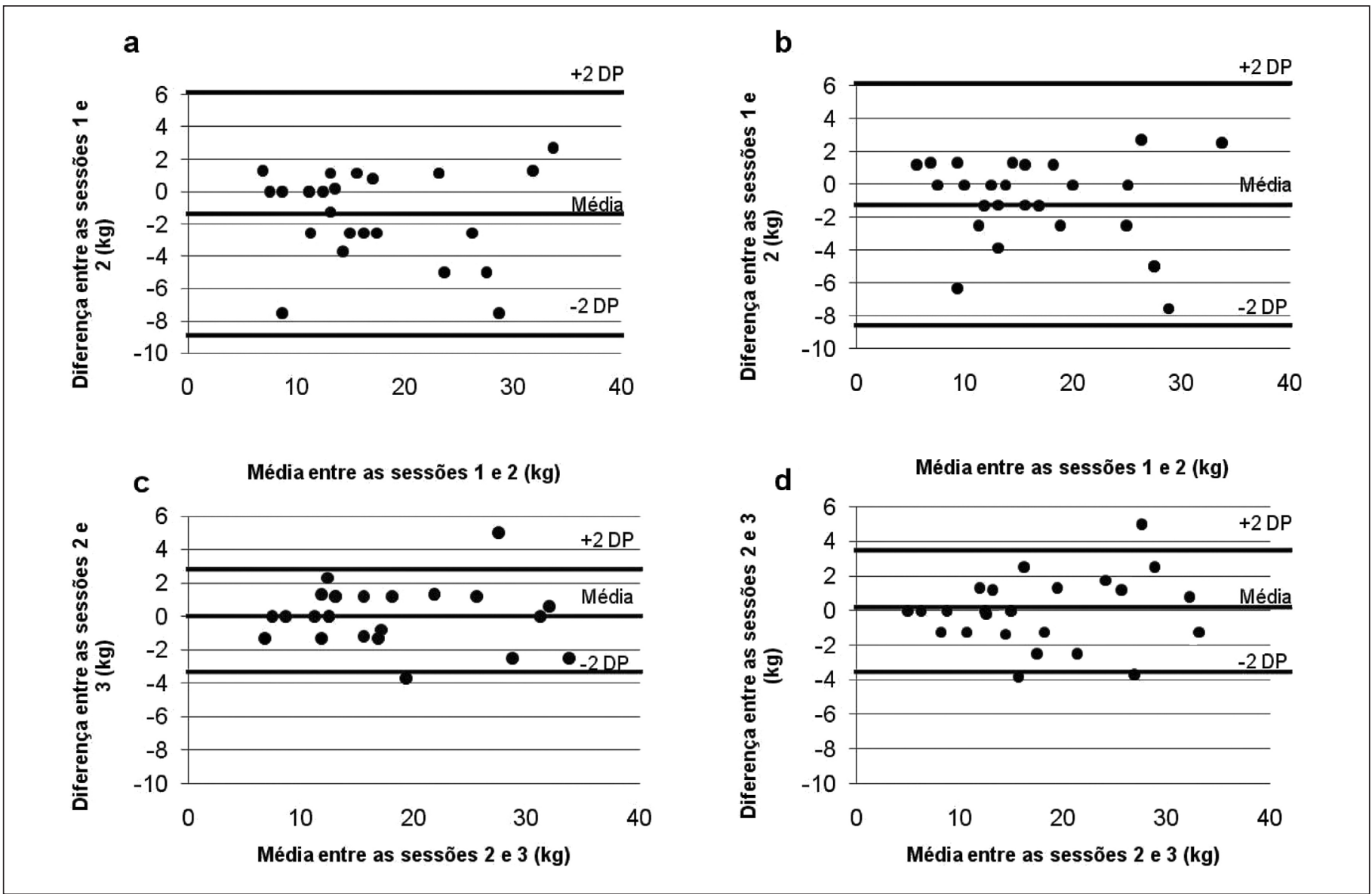

Figura 1. Diferença média e limites de concordância da carga de 1-RM entre as sessões 1 e 2 ( $a$ - perna com menor ITB e b - perna com maior ITB) e entre as sessões 2 e 3 (c - perna com menor ITB e d - perna com maior ITB). 
Os resultados do presente estudo estão de acordo com os de estudos anteriores ${ }^{(10,19,20)}$, que têm demonstrado em diferentes populações a necessidade de realização de mais de uma sessão de teste de 1-RM para a obtenção de resultados acurados de força máxima. O número de sessões necessárias para a obtenção da carga máxima com o teste de 1-RM tem sido amplamente discutido na literatura, uma vez que alguns estudos indicaram a necessidade de realização de até nove sessões de teste de 1-RM. No presente estudo, a carga de 1-RM aumentou da primeira para a segunda sessão de teste, e não aumentou da segunda para as demais sessões, sugerindo que duas sessões de testes são suficientes para obtenção de medidas acuradas da força máxima. Esses resultados corroboram com estudos anteriores que também verificaram a necessidade de realizar duas sessões de teste de 1-RM para a obtenção acurada da carga máxima em indivíduos idosos ${ }^{(19,20)}$.

Os mecanismos envolvidos no aumento de força entre as sessões de teste de 1-RM não foram investigados neste estudo, bem como em estudos anteriores. Entretanto, tem sido sugerido que as adaptações neurais, que geralmente são observadas nas fases iniciais de um programa de treinamento de força, estariam relacionadas com esses aumentos. Assim, fatores de ordem neural, como por exemplo, a maior ativação muscular, aumento da frequência de disparo das unidades motoras e alterações na excitabilidade do motoneurônio, estariam envolvidos nesta resposta.

Embora tenha havido uma tendência $(P=0,10)$, a força máxima foi similar entre os membros com menor e com maior ITB. Como a DAOP está associada à atrofia muscular ${ }^{(1)}$, denervação e neuropatia periférica ${ }^{(21)}$, era esperado que o membro em que a doença é mais grave (com menor ITB) apresentasse menores níveis de força máxima. Contudo, é importante observar que, embora a força muscular tenha sido avaliada no membro com maior e menor gravidade da doença, a maioria dos indivíduos apresentava a doença nos dois membros. De fato, o valor médio do ITB do membro com maior ITB foi 0,72, que corresponde a um valor de diagnóstico da DAOP $(\mathrm{ITB}<0,90)$. Consequentemente, se os indivíduos incluídos no presente estudo apresentassem a doença em um membro, é possível que diferenças entre os membros pudessem ter sido evidenciadas.

Um aspecto importante do presente estudo é que não foram observadas lesões ou eventos cardiovasculares durante os testes de
1-RM nos indivíduos com DAOP. Essa observação está de acordo com estudos anteriores que têm demonstrado baixas taxas de lesão durante o teste de 1-RM em idosos ${ }^{(22,23)}$. Além disso, vale ressaltar que estudos têm indicado que o teste de 1-RM promove baixa sobrecarga cardiovascular ${ }^{(24)}$, o que é um ponto importante a ser considerado em indivíduos com DAOP, que apresentam risco cardiovascular aumentado(25). Por outro lado, é importante considerar que os indivíduos deste estudo passaram por triagem quanto a problemas ortopédicos e cardiovasculares, o que, sem dúvida, minimizou a chance de ocorrência de eventos adversos.

Este estudo apresenta algumas limitações importantes. Primeiro, foi utilizado apenas um exercício e o modo de contração utilizado foi isoinercial. Consequentemente, a extrapolação dos resultados para outros exercícios ou outras formas de contração (isocinética ou isométrica) é limitada. Segundo, sabe-se que o local mais frequente dos sintomas de claudicação intermitente são os músculos posteriores de perna. Entretanto, como a reprodutibilidade dos testes de força nos músculos posteriores da perna é menor do que no músculo do quadríceps em indivíduos com DAOP(2), foi feita a opção pelo exercício extensão de joelhos, uma vez que a força de quadríceps têm sido associada a limitação funcional de idosos ${ }^{(26,27)}$ e com a gravidade da $\mathrm{DAOP}^{(3,28)}$. Finalmente, a amostra foi composta exclusivamente por indivíduos com DAOP e sintomas de claudicação intermitente, o que limita a extrapolação dos resultados para outros níveis da DAOP também é limitada.

\section{CONCLUSÃO}

A medida acurada da força máxima usando o teste de 1-RM, no exercício extensão de joelhos, em indivíduos com DAOP e sintomas de claudicação intermitente, é obtida quando duas sessões de testes de 1-RM são realizadas.

\section{AGRADECIMENTOS}

Esse estudo recebeu apoio da Fundação de Amparo à Pesquisa do Estado de São Paulo (processo 06/003759-3).

Todos os autores declararam não haver qualquer potencial conflito de interesses referente a este artigo.

\section{REFERÊNCIAS}

1. Regensteiner JG, Wolfel EE, Brass EP, Carry MR, Ringel SP, Hargarten ME, et al. Chronic changes in skeletal muscle histology and function in peripheral arterial disease. Circulation 1993:87:413-21.

2. Scott-Okafor HR, Silver KK, Parker J, Almy-Albert T, Gardner AW. Lower extremity strength deficits in peripheral arterial occlusive disease patients with intermittent claudication Angiology. 2001;52:7-14.

3. McDermott MM, Criqui MH, Greenland P, Guralnik JM, Liu K, Pearce WH, et al. Leg strength in peripheral arterial disease: associations with disease severity and lower-extremity performance. J Vasc Surg 2004;39:523-30.

4. Atkins LM, Gardner AW. The relationship between lower extremity functional strength and severity of peripheral arterial disease. Angiology 2004;55:347-55.

5. Kuo HK, Yu YH. The relation of peripheral arterial disease to leg force, gait speed, and functional dependence among older adults. J Gerontol A Biol Sci Med Sci 2008;63:384-90.

6. McGuigan MR, Bronks R, Newton RU, Sharman MJ, Graham JC, Cody DV, et al. Resistance training in patients with peripheral arterial disease: effects on myosin isoforms, fiber type distribution, and capillary supply to skeletal muscle. J Gerontol A Biol Sci Med Sci 2001;56:B302-10.

7. Wang J, Zhou S, Bronks R, Graham J, Myers S. Effects of supervised treadmill-walking training on strength and endurance of the calf muscles of individuals with peripheral arterial disease. Clin Sport Med 2006:16:397-400

8. Hopkins WG. Measures of reliability in sports medicine and science. Sports Med 2000;30:1-15.

9. De Vos NJ Singh NA, Ross DA, Stavrinos TM, Orr R, Fiatarone Singh MA. Continuous hemodynamic response to maximal dynamic strength testing in older adults. Arch Phys Med Rehabil 2008;89:343-50.

10. Ploutz-Snyder LL, Giamis EL. Orientation and familiarization to $1 \mathrm{RM}$ strength testing in old and young women. J Strength Cond Res 2001;15:519-23.

11. Norgren L, Hiatt WR, Dormandy JA, Nehler MR, Harris KA, Fowkes FG, et al. Inter-society consensus for the management of peripheral arterial disease. Int Angiol 2007;26:81-157.

12. Gardner AW, Skinner JS, Cantwell BW, Smith LK. Progressive vs single-stage treadmill tests for evaluation of claudication. Med Sci Sports Exerc 1991;23:402-8.

13. Fontaine R, Kim M, Kieny R. [Surgical treatment of peripheral circulation disorders.]. Helv Chir Acta 1954;21:499-533.

14. 2003 European Society of Hypertension-European Society of Cardiology guidelines for the management of arterial hypertension. J Hypertens 2003:21:1011-53.
15. Enoka RM. Neural adaptations with chronic physical activity. J Biomech 1997;30:447-55.

16. Matuszak ME, Fry AC, Weiss LW, Ireland TR, McKnight MM. Effect of rest interval length on repeated 1 repetition maximum back squats. J Strength Cond Res 2003;17:634-7.

17. Clarke DH. Adaptations in strength and muscular endurance resulting from exercise. Exerc Sport Sci Rev 1973;1:73-102

18. Rydwik E, Karlsson C, Frandin K, Akner G. Muscle strength testing with one repetition maximum in the arm/shoulder for people aged 75 + - test-retest reliability. Clin Rehabil 2007;21:258-65.

19. Levinger I, Goodman C, Hare DL, Jerums G, Toia D, Selig S. The reliability of the 1RM strength test for untrained middle-aged individuals. J Sci Med Sport 2009;12:310-6.

20. Phillips WT, Batterham AM, Valenzuela JE, Burkett LN. Reliability of maximal strength testing in older adults. Arch Phys Med Rehabil 2004;85:329-34.

21. England JD, Regensteiner JG, Ringel SP, Carry MR, Hiatt WR. Muscle denervation in peripheral arterial disease. Neurology 1992;42:994-9.

22. Kaelin ME, Swank AM, Adams KJ, Barnard KL, Berning JM, Green A. Cardiopulmonary responses, muscle soreness, and injury during the one repetition maximum assessment in pulmonary rehabilitation patients. Journal of Cardiopulmonary Rehabilitation 1999;19:366-72.

23. Shaw CE, McCully KK, Posner JD. Injuries during the one repetition maximum assessment in the elderly J Cardiopulm Rehabil 1995;15:283-7.

24. Nery SS. Pressão arterial de hipertensos estágio I durante diferentes intensidades de exercício resistido. São Paulo: Universidade de São Paulo; 2005.

25. Steg PG, Bhatt DL, Wilson PW, D'Agostino R, Sr., Ohman EM, Rother J, et al. One-year cardiovascular event rates in outpatients with atherothrombosis. JAMA 2007;297:1197-206.

26. Rantanen T, Avlund K, Suominen H, Schroll M, Frandin K, Pertti E. Muscle strength as a predictor of onset of ADL dependence in people aged 75 years. Aging Clin Exp Res 2002;14:10-5.

27. Ploutz-Snyder LL, Manini T, Ploutz-Snyder RJ, Wolf DA. Functionally relevant thresholds of quadriceps femoris strength. J Gerontol A Biol Sci Med Sci 2002;57:B144-52.

28. McDermott MM, Tian L, Ferrucci L, Liu K, Guralnik JM, Liao Y, et al. Associations between lower extremity ischemia, upper and lower extremity strength, and functional impairment with peripheral arteria disease. J Am Geriatr Soc 2008;56:724-9. 